\title{
Operation for repair of obstructed substernocostal (Morgagni) hernia
}

\author{
K MOGHISSI
}

From Humberside Cardiothoracic Surgical Centre, Castle Hill Hospital, Cottingham, North Humberside

ABSTRACT A complicated case of large substernocostal hernia in a 67-year-old man is described. A new surgical approach via upper laparotomy which can be extended by a lower median sternotomy, allowing complete access to the hernia was tried successfully and the patient's recovery was uneventful.

A hernia occurring through a retrosternal defect first described by Morgagni in 1769 has received many names, among which that of substernocostal hernia ${ }^{1}$ appears more appropriate. The embryology and anatomical variations of this type of hernia have been fully reported. ${ }^{2-4}$ It has been pointed out $^{135}$ that these herniae are rare and many do not require repair. For those patients requiring operation, abdominal, ${ }^{2}{ }^{3}$ thoracic, ${ }^{2}$ or substernal ${ }^{4}$ approaches may be used according to the experience of the operator, and the anatomical variations of the herniae. In six previous cases we undertook the repair through a thoracotomy in three patients and a laparotomy in the other three with relative ease.

In a particularly complicated case such as the one reported below these approaches may prove inadequate and arduous. This paper concerns a new surgical approach and operation (abdominomediastinal) for such herniae.

\section{Case report}

A 67-year-old man was transferred to our unit from another hospital as an emergency because of vomiting, haematemesis, and melaena for two to three days. He was thought to have a "Morgagni" hernia the exact details of which were not known. On admission the patient was shocked with signs of upper alimentary tract obstruction. Initially, he was treated by anti-shock therapy, blood transfusion, and naso-gastric tube suction for decompression.

Full investigations including chest radiograph, endoscopic examination, and barium swallow confirmed the diagnosis of obstructed substernocostal

Address for reprint requests: Mr K Moghissi, Humberside Cardiothoracic Surgical Centre, Castle Hill Hospital, Cottingham, North Humberside. hernia containing the stomach, the first part of duodenum, and some small bowel loops. An emergency operation was planned.

\section{Operation}

The patient was placed in the supine position. A laparotomy was carried out through an upper midline incision. This confirmed the diagnosis of a substernocostal hernia with a sac containing the upper abdominal viscera protruding into the anterior mediastinum, the left chest, and predominantly the right chest. A complete assessment of the hernia and its contents was impossible because of the relatively narrow opening of the defect. Even incising the edges of the neck of the sac did not allow reduction of the sac or the contents. The skin incision was therefore extended upwards over the xiphoid process and the lower part of the body of the sternum. A median sternotomy was carried out over the same extent. This allowed perfect exposure of the sac, which was attached to the pericardium and the base of the right lung. The sac was opened from above and the neck incised. There were multiple firm adhesions between the contents and the sac on one hand, and between the viscera within the sac on the other, producing mesenteric oedema and congestion.

The contents of the sac consisted of the whole of 0 the stomach rotated on its horizontal axis, the transverse colon, the gastro-colic omentum, the $\stackrel{\mathscr{C}}{-}$ greater omentum, and loops of small intestine. The $\frac{T}{\sigma}$ viscera within the sac could be reduced into the abdomen after dividing adhesions. The substerno- $\cong$ costal defect (fig 1) was $12 \mathrm{~cm}$ long and $4 \mathrm{~cm}$ deep, $\stackrel{\mathbb{D}}{\Omega}$ extending from $3 \mathrm{~cm}$ to the left of the midline to $\bar{\sigma}$ $8-9 \mathrm{~cm}$ to the right of the midline. 

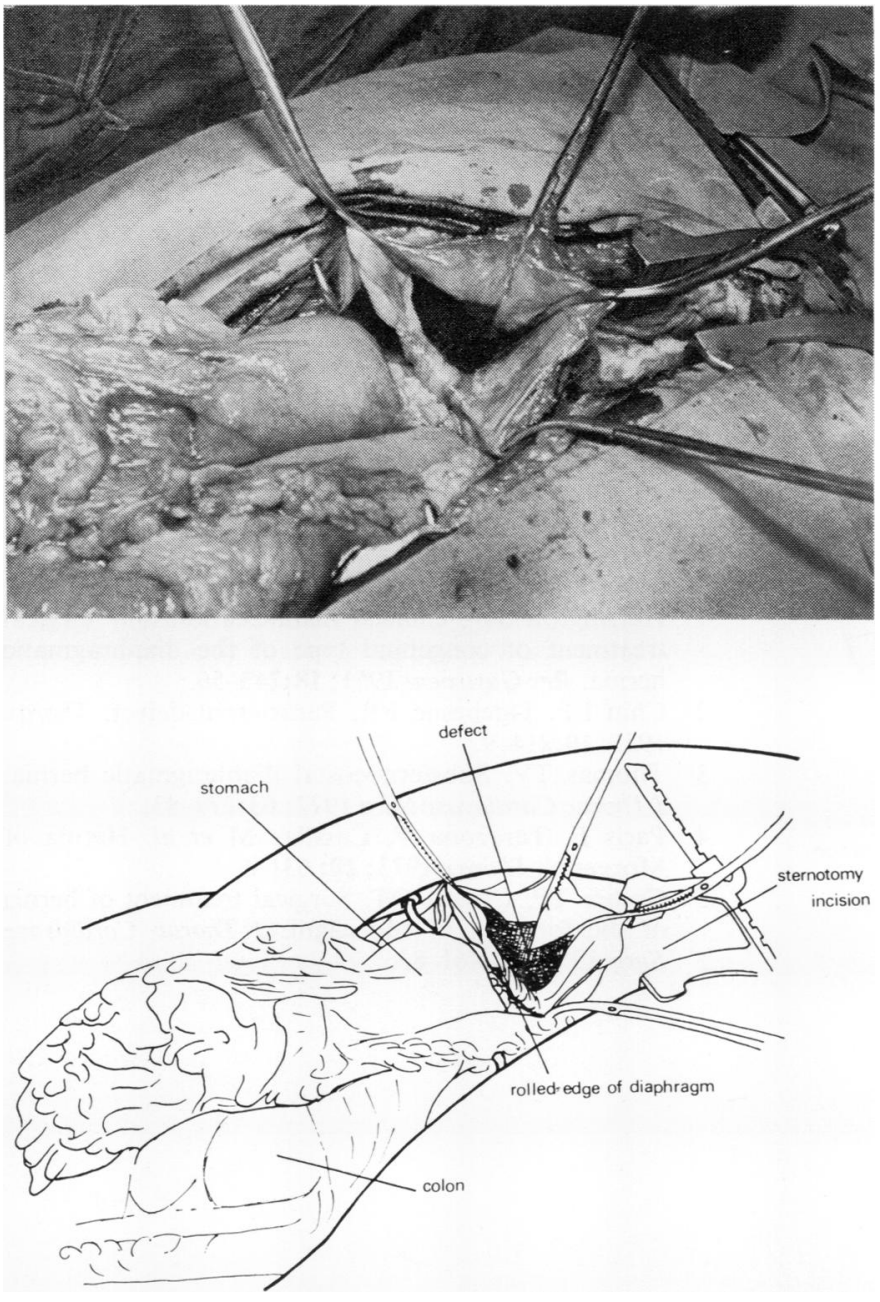

Fig 1 Defect after reduction of the hernia.

Before the repair of the defect the base of the right lung was decorticated, the sac partially excised and the right pleural space drained.

Repair of the defect was carried out by approximating the rolled edge of the diaphragm, forming the posterior boundary of the defect, to the costal margin laterally, and the sternum medially (the anterior boundary of the defect) using no 1 silk and wire stitches (fig 2). Medially the wire stitches were inserted by entering through one half of the divided sternum, picking up the rolled edges of the diaphragm reinforced by Teflon patches, and then going through the second half of the sternum. Three such stitches were inserted but left untied until the lateral part of the repair of the defect was completed. These three wire stitches were then tied approximating the two halves of the sternum on the one hand, and the diaphragm to the sternum on the other. The abdominal and pre-sternal wounds were then closed.

The patient's recovery was uneventful. He was discharged two weeks after the operation completely asymptomatic.

We suggest that the approach to larger substernocostal herniae, particularly if they are complicated, should be an upper midline laparotomy which can be extended by a lower median sternotomy, thus allowing complete access to the hernia.

We are grateful to Mrs FM Strugnell for her help in the presentation of this manuscript and the illustrations, and to Mrs C Scott for secretarial assistance. 


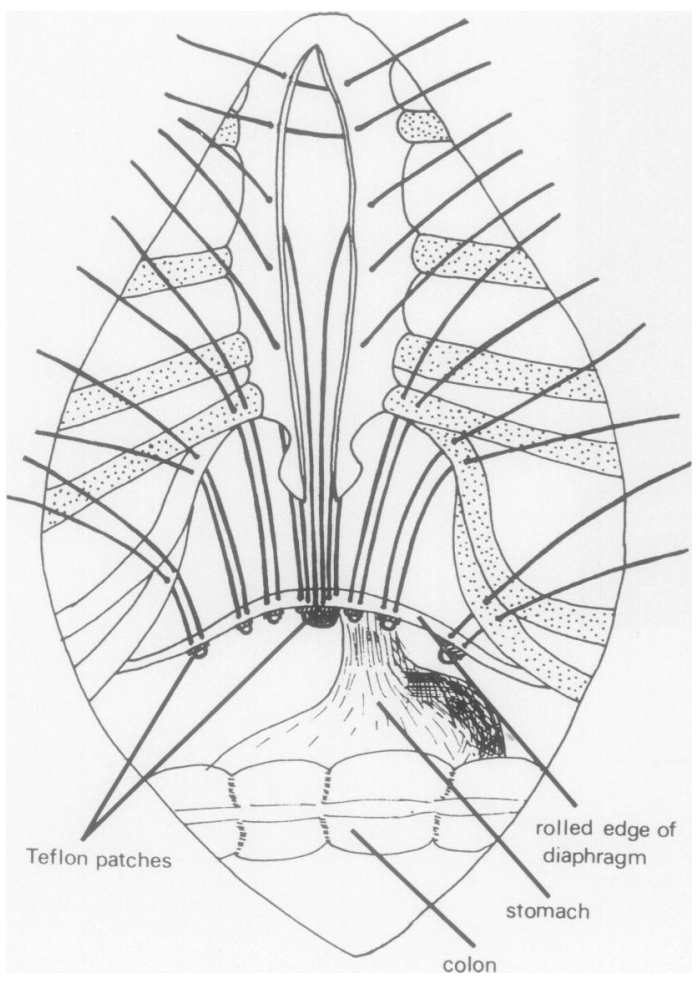

Fig 2 Position of the stitches approximating the rolled edge of the diaphagm to the costal margin and the sternum.

\section{References}

1 Harrington SW. Clinical manifestation and surgical treatment of congenital type of the diaphragmatic
hernia. Rev Gastroent 1951; 18:243-56.

2 Chin EF, Duchesne ER. Parasternal defect. Thorax $\vec{\bullet}$ 1955; 10:214-9.

3 Thomas TV. Substernocostal diaphragmatic hernia. J Thorac Cardiovasc Surg 1972; 63:279-83.

4 Paris F, Tarazona V, Cassilas M et al. Hernia of Morgagni. Thorax 1973; 28: 631-6.

5 Comer TP, Clagett OT. Surgical treatment of hernia of the foramen of Morgagni. $J$ Thorac Cardiovasc Surg 1965; 52:461-8. 